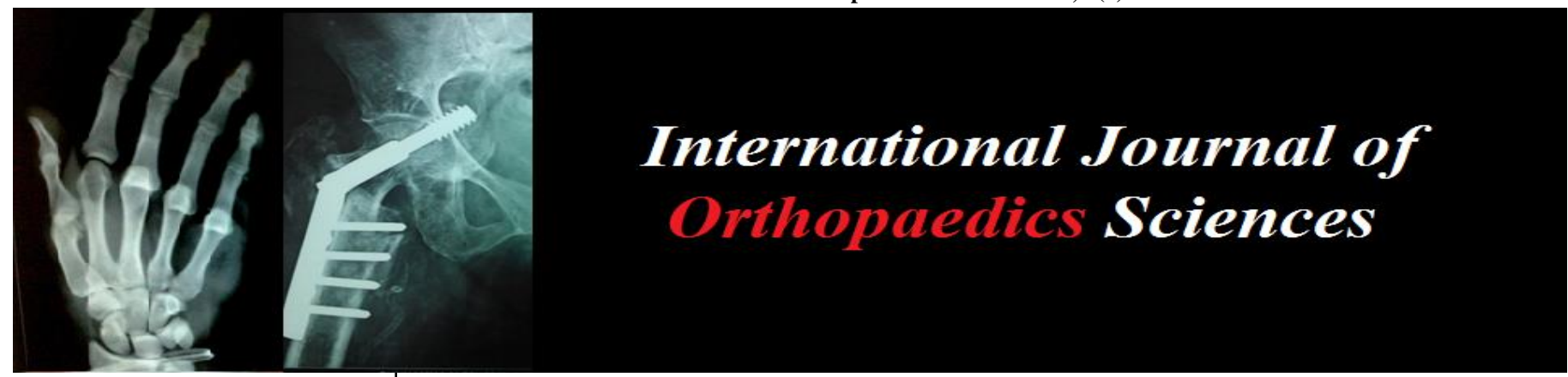

E-ISSN: 2395-1958

P-ISSN: 2706-6630

IJOS 2020; 6(4): 85-89

(C) 2020 IJOS

www.orthopaper.com

Received: 18-06-2020

Accepted: 22-07-2020

Dr. Amol Gowaikar

Assistant Professor, Department

of Orthopaedic, Dr. D.Y. Patil

Hospital, Kolhapur,

Maharashtra, India

Dr. Ravindra Patil

Associate Professor, Department of Orthopaedic, Dr. D.Y. Patil

Hospital, Kolhapur,

Maharashtra, India

Dr. Shreyansh Mehta

2nd year Resident, Department of Orthopaedic, Dr. D.Y. Patil

Hospital, Kolhapur,

Maharashtra, India

Dr. Vijaysinh Manik Bhosale 3rd year Resident, Department of Orthopaedic, Dr. D.Y. Patil Hospital, Kolhapur,

Maharashtra, India

Dr. Akshay Sanjay Anchalia 3rd year Resident, Department of Orthopaedic, Dr. D.Y. Patil Hospital, Kolhapur,

Maharashtra, India

Dr. Yash P Oza

2nd year Resident, Department of Orthopaedic, Dr. D.Y. Patil

Hospital, Kolhapur,

Maharashtra, India
Corresponding Author: Dr. Amol Gowaikar Assistant Professor, Department of Orthopaedic, Dr. D.Y. Patil Hospital, Kolhapur,

Maharashtra, India

\section{To study the clinical outcome in distal humerus fractures with orthogonal plating via the olecranon osteotomy approach}

Dr. Amol Gowaikar, Dr. Ravindra Patil, Dr. Shreyansh Mehta, Dr. Vijaysinh Manik Bhosale, Dr. Akshay Sanjay Anchalia and Dr. Yash P Oza

DOI: https://doi.org/10.22271/ortho.2020.v6.i4b.2323

\section{Abstract}

Objective: The aim of our study was to evaluate the clinical outcome of distal humerus fractures treated with orthogonal plating via the olecranon osteotomy approach in the Indian population, at a tertiary care centre in Kolhapur.

Method: A prospective study was carried out in a tertiary care center in Kolhapur. A total number of 16 patients with fractures of distal humerus (AO type B and C) were studied between May2019 to August 2020. All were treated with orthogonal plating via the olecranon osteotomy approach. A mean follow up of 6 months was carried out, and evaluation was done with the help of MEPS and DASH scoring system. Result: The mean radiological union time was 14.6 weeks with an arc of flexion of 101.2 degrees. The outcome measured using Mayo Elbow Performance Score (MEPS) and DASH scoring was 89 and 15.4 points respectively at 6 months. Patient satisfaction was $87 \%$ at the end of 6 month follow-up.25\% patients reported complications like infection (1 patient), delayed wound healing ( 2 patients) and hardware prominence (1 patient). No cases of infection or peripheral neuropathies were reported in any of the patients.

Conclusion: Orthogonal plating via the olecranon osteotomy approach remains a good alternative showing good clinical outcome in the treatment of distal humerus fractures (AO type B and C). It offers a more stable fixation and the olecranon osteotomy approach gives better exposure for surgery.

Keywords: Distal humerus, intra-articular fracture, orthogonal plating, olecranon osteotomy approach

\section{Introduction}

Distal Humerus fractures account for 2-6\% of all fractures and comprises of approximately a one-third of fractures around the elbow. The mechanism of injury is generally a high velocity trauma and therefore, may have a complex presentation ${ }^{[2]}$. In elderly it is due to a result of domestic falls, generally resulting in intra-articular or bi-columnar fracture of humerus ${ }^{[3]}$. As per the AO classification, supracondylar fractures of humerus can be classified as extraarticular (AO type A), partially articular (AO type B), or completely articular (AO type C) ${ }^{[4]}$. The Jupiter and Mehne classification system is also utilized which is based on the fracture patterns, allowing better preoperative planning ${ }^{[5]}$.

The distal humerus is composed of a medial and lateral column with a central area of thin weaker bone. This central area forming the coronoid and olecranon fossae, is present to facilitate elbow flexion and extension by allowing a space for the olecranon tip to articulate. It also provides bony stability. However, this central area has been shown to be particularly thin in patients with osteopenia thus making it a common site of involvement with fractures of the distal humerus ${ }^{[6]}$. It is essential to reconstruct anatomy to restore diaphyseal-metaphyseal contact in order to provide the most stability. This allows the best healing potential ${ }^{[7]}$. Poor treatment outcomes in this highly mobile joint requires personalisation of treatment protocols in order to get the most satisfactory clinical outcome.

Distal Humerus fractures were traditionally treated with multiple Kirschner wire fixations or by conservative methods. This resulted in high incidence of severe elbow stiffness, and 
malunion among other complications causing loss of function. This method cannot be used in elderly patients with severe medical co-morbiditiesor those unfit for anaesthesia.

Therefore, the current trend of management for distal humerus fractures is Open Reduction and Internal Fixation (ORIF) with bicolumnar plating to achieve anatomical reduction, maintainjoint congruity, maintain stable fixation of elbow joint and avoidance of complications ${ }^{[7,9]}$.

In cases where it is difficult to do ORIF, like for fractures with significant comminution or in low lambda type (Mehne classification), it is suggested that Total Elbow Arthroplasty is a better alternative ${ }^{[10]}$. However, a recent meta-analysis has shown similar clinical outcomes with respect to Total Elbow Arthroplasty and ORIF.

Taking into account the unique anatomy of the distal end of humerus, various types of implants have been used. The implants must be strong enough to withstand the bending forces until the union of fracture fragments is achieved. Hence, in our study a standardised $3.5 \mathrm{~mm}$ pre-contoured locking and non-locking plates were used.

The anatomical placement of plates is highly debated as to the position of the plates to achieve maximum stability and maintain anatomical reduction. Orthogonal (90-90) plating or perpendicular plating involves placement of one plate on the medial column and another over the posterolateral column. This results in better stability and maintaining anatomical reduction.

\section{Aim}

The aim of our study was to evaluate the clinical outcome of distal humerus fractures treated with orthogonal plating via the olecranon osteotomy approach in the Indian population at a tertiary care centre in Kolhapur.

\section{Materials and Methods}

This is a prospective study done from May 2019 to August 2020, in 16patients of distal humerus fractures with AO type $\mathrm{B}$ and $\mathrm{C}$. All patients were treated with orthogonal plating via the olecranon osteotomy approach in a tertiary care centre in Kolhapur. All patients included in our study were between 18 to 60 years of age. Simple Random method was used for the study as sampling method.

\section{Inclusion Criteria}

- All Patients with AO Classification AO Type B and C.

- Closed fractures and Gustilo Anderson classification upto Type 2.

- All patients with pre-anaesthetic fitness.
- Patients below 18 years of age and above 60 years of age.

- All compound fractures with Gustilo-Anderson Type 3.

- Patients with prior surgery around the elbow joint.

- Patients with prior ulnar neuropathy.

Plain radiographs of the concerned elbow with AP (anteroposterior) and Lateral views were taken along with CT of the concerned elbow with $3 \mathrm{D}$ reconstruction. All the necessary investigations for pre-operative anaesthetic fitness were also done.

Written informed consent was taken from all patientsprior to surgery.

The Mayo Elbow Performance Score (MEPS) and Disability of Arm Shoulder and Hand Score (DASH) were used as evaluation criteria for the calculation of functional outcome of the patient.

\section{Surgical Technique}

All 16 Patients were operated at a tertiary care center in Kolhapur. All patients were operated under regional anaesthesia. Pneumatic Tourniquet was used in all cases. All patients were operated in lateral position with side arm attachment.

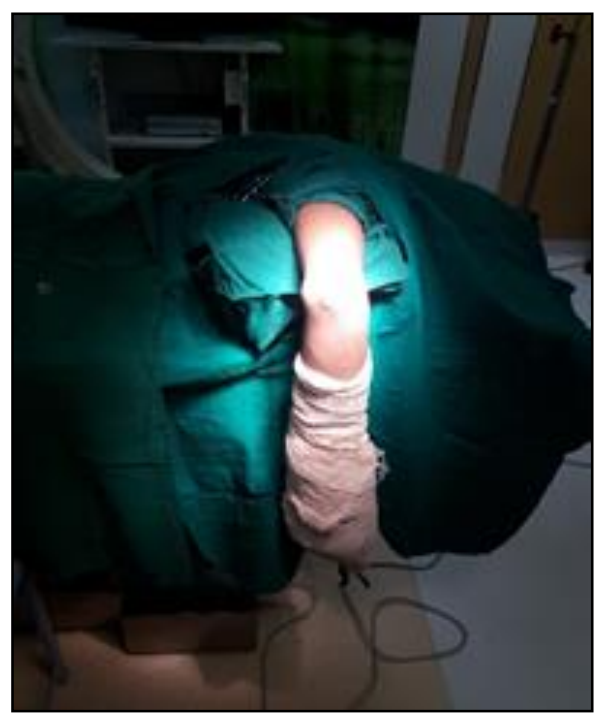

Fig 1: Lateral Position with side arm

Elbow was exposed posteriorly through a midline incision of about $15 \mathrm{~cm}$ beginning proximal to the tip of olecranon and extending distally towards the forearm with slight radial deviation. In all cases ulnar nerve was isolated, tagged and retracted to avoid any damage.

\section{Exclusion Criteria}
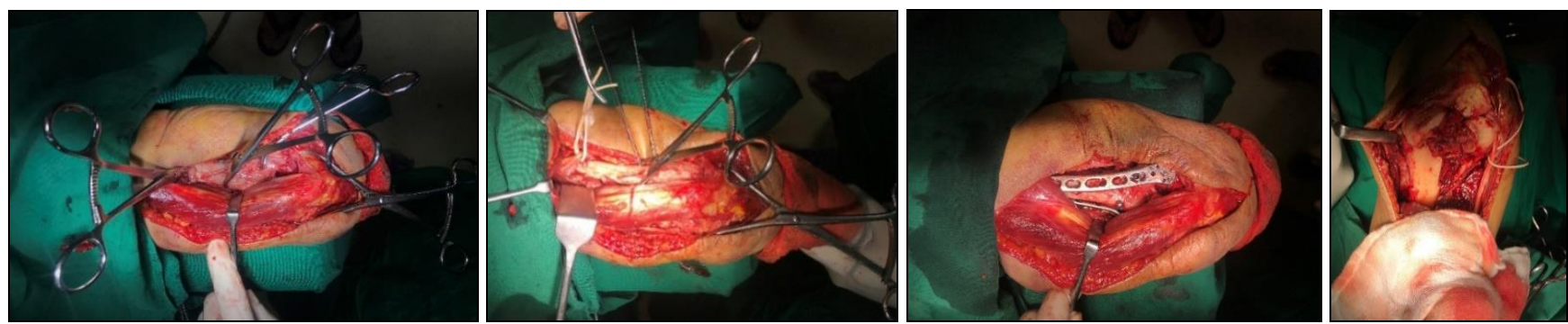

Fig 2: Intra operative images

In all cases intra articular Chevron Osteotomy was performed to expose the joint with help of a thin osteotome and multiple drill holes. The olecranon process along with the insertion of triceps muscle was elevated proximally. Fracture was reduced with help of Kirchner wires and bone reduction clamps under image intensifier guidance. Medial and lateral columns were 
reconstructed with the help of $3.5 \mathrm{~mm}$ precontoured locking plates and appropriate screws.

Intraoperatively, elbow joint was checked for stability and for anatomical reduction.

Olecranon osteotomy was fixed with Tension Band wiring and closure was done in layers with suction drain no. 12, kept and removed post-operatively on the 2 nd day. All patients were given an above elbow slab with arm sling for upto 3 weeks. Limb elevation was given for 48 hours in the postoperative period to reduce swelling. Intravenous antibiotic coverage and analgesics were given till post-operative day 2 and thereafter only oral analgesics were continued until day 5 . Suture removal was done on $14^{\text {th }}$ day post-operatively. Physiotherapy for intermittent mobilisation was started from day 5 onwards, while continuing with the above elbow slab. Aggressive physiotherapy was started after 3 weeks postoperatively.

\section{Results}

A total of 17 patients were included in the study. One patient was lost to follow up. Of the remaining 16 patients, the mean age of the patient was 40 years. 10 patients $(63 \%)$ were males, and 6 patients $(37 \%)$ were females. Right sided involvement (13 patients) was more frequent in the study than left side involvement (3 patients). 12 patients $(75 \%)$ sustained the fracture due to road traffic accidents and4 patients $(25 \%)$ had a history of domestic fall. All the fractures were classified using the AO Classification system. 4 patients (25\%) were AO type B whereas 12 patients $(75 \%)$ were AO type $\mathrm{C}$ fractures respectively.

The mean time taken between onset of injury and surgical intervention was 4.1 days. The average duration of surgery was 94 minutes. There were no cases of neurovascular injury encountered in the present study. The average duration of the radiological union was 14 weeks in $10(60 \%)$ cases, 15 weeks in $4(25 \%)$ cases, 19 weeks in $2(15 \%)$ cases.

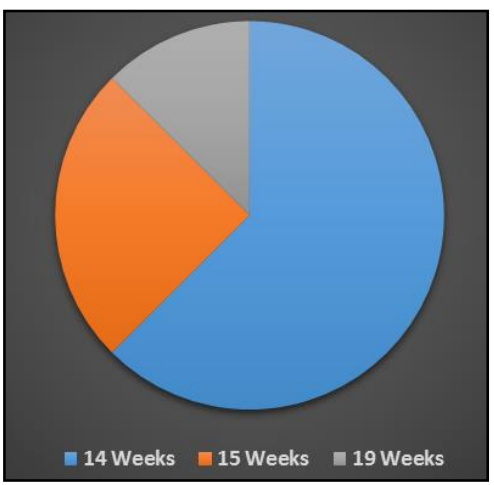

Fig 3: Union Time

Hardware prominence was seen in $1(6.25 \%)$, infection in $1(6.25 \%)$ and delayed wound healing $2(13 \%)$ cases. The infection was in the superficial plane and was treated with daily dressing and the appropriate antibiotics after isolating the organism by pus culture and sensitivity method. There was no recurrence of infection in that case. There were no cases of deep infection encountered in the present study. No cases of non-union or malunion were observed. None of the patients in the present study required the above elbow slab after 3 weeks post-operatively. 3 (19\%) patients started physiotherapy after 4 weeks post-operatively due to poor compliance.

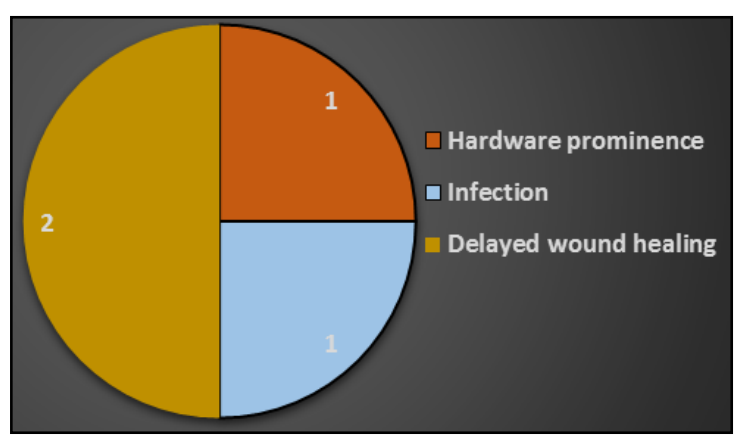

Fig 4: Complication

All the patients were followed-up at 3 weeks, 3 months and 6 months post-operatively. The outcome was calculated using the MEPS and DASH scores. The MEPS score showed excellent result in $3(19 \%)$, good in $10(62 \%)$, fair in $3(19 \%)$ and no poor outcome. The mean DASH score was 15.4 in patients. None of the patients presented with failure of fixation of the olecranon osteotomy.

Out of 16 patients, 9 cases had excellent outcome with nearly complete range of motion at 3 month follow up and almost no to mild pain and a stable joint, 5 patients had good outcome with average arc of motion 100(range 45-135) with mild to moderate pain and a stable joint, in 2 case fair result was obtained with only 70 degree of flexion, moderate pain and relatively unstable joint due to poor bone stock and compliance to physiotherapy.

Table 1: Dash score at the end of 6 month

\begin{tabular}{|c|c|}
\hline $0-20$ & 12 \\
\hline $21-40$ & 4 \\
\hline $41-60$ & 0 \\
\hline $61-80$ & 0 \\
\hline $81-100$ & 0 \\
\hline
\end{tabular}

MEPS Score At 6 Month

\begin{tabular}{|c|c|r|}
\hline Excellent & 9 & $56.25 \%$ \\
\hline Good & 5 & $31.25 \%$ \\
\hline Fair & 2 & $12.50 \%$ \\
\hline
\end{tabular}

\begin{tabular}{|c|c|c|}
\hline Variable & Number & $\%(n=16)$ \\
\hline \multicolumn{3}{|c|}{ Sex } \\
\hline Male & 10 & 63 \\
\hline Female & 6 & 37 \\
\hline \multicolumn{3}{|c|}{ Side } \\
\hline Right & 13 & 81 \\
\hline Left & 3 & 19 \\
\hline \multicolumn{3}{|c|}{ Mode of injury } \\
\hline Road Traffic Accident & 12 & 75 \\
\hline Domestic fall & 4 & 25 \\
\hline \multicolumn{3}{|c|}{ AO Classification } \\
\hline $12-\mathrm{B}$ & 4 & 25 \\
\hline $12-\mathrm{C}$ & 12 & 75 \\
\hline \multicolumn{3}{|c|}{ Union } \\
\hline 14 Weeks & 10 & 62 \\
\hline 15 Weeks & 4 & 25 \\
\hline 19 Weeks & 2 & 13 \\
\hline
\end{tabular}




\section{Discussion}

The distal humerus is a triangular structure represented by the medial and lateral columns with the intervening articular surface ${ }^{[14]}$ and thus, the stability depends upon this system. The ORIF method remains the standard of care in the treatment of intra-articular distal humerus fractures in the physiologically active patient ${ }^{[15,16,17]}$. The treatment of distal humeral fractures is complex, and expectedly, there is a high incidence of complications ${ }^{[18]}$. Severe comminution, bone loss, and osteopenia, predispose distal humeral fractures to unsatisfactory results due to inadequate fixation ${ }^{[19,20]}$

The utilization of two plates, one on the medial column and the other on the lateral column ${ }^{[21]}$ is required for recreation of a stable anatomical joint. Orthogonal plating techniques evolved after a publication by Jupiter and colleagues in 1985, reported on patients having successful outcomes with ORIF of distal humerus fractures [21]. They noted that the key to surgical success was obtaining complete bony stability to permit early range of motion. It became accepted that Kirschner wire fixation alone did not provide adequate stability to treat bicolumnar distal humeral fractures ${ }^{[22,23]}$. A high incidence of post-operative intra-articular step $>1 \mathrm{~mm}$ and angulations of $>5$ degrees was observed in cases treated with CRIF with Kirschner wires. In addition, Waddell and colleagues have shown that elbow immobilization of three to four weeks post-operatively leads to unacceptable stiffness ${ }^{[24,}$ ${ }^{28]}$. In contrary to the study by Kumar et al. ${ }^{[25]}$ where a 4.5 mm single LCP was used with 4 cortical screw purchase for fixation, we used a combination of $23.5 \mathrm{~mm}$ distalhumerus locking platespositioned in 90 degrees or orthogonal configuration. However, studies by Shin et al. in 2010 and Lan et al. in 2013 reported no difference in outcomes between parallel and perpendicular positioning of the plates ${ }^{[26,27]}$. This was contrary to Joshua M. Abzug et al. ${ }^{[28]}$ who concluded that parallel plating may be the preferred technique for very distal humerus fracture patterns since more stability can be obtained by providing additional screws in the distal fragment. Parallel plating also offers more compression at the fracture site. ${ }^{[27]}$. It further stated that orthogonal plating may be preferred over parallel plating in cases of an anterior shear fracture where the fixation from posterior to anterior will provide additional stability to the intra-articular fracture fragments. We found that orthogonal plating was a better method for maintaining reduction owing to its triangular framework.

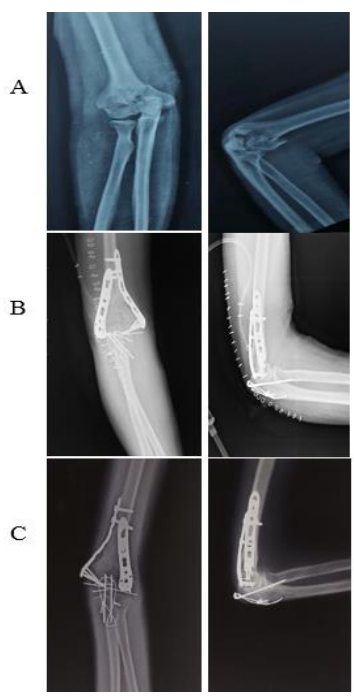

Fig 3: Radiograph of elbow AP and LAT view (A) Pre- Operative $x$ ray (B) Immediate x-ray (C) 6 Weeks

\section{Follow up}

The elbow joint is a highly mobile joint offering flexionextension from 0-135 degrees. The main complications around the elbow joint are infection, delayed wound healing and hardware prominence ${ }^{[12]}$. Use of NSAIDs and strict limb elevation in the immediate post-operative period kept the incidence of infection in the present study in line with the already published literature. A large incision with large amount of soft tissue dissection and handling causes delayed wound healing due to decrease in localised vascularity. However, adequate visualisation due to the olecranon osteotomy approach decreased soft tissue handling and kept the incidence of delayed wound healing within acceptable limits. Hardware prominence decreases patient satisfaction and may cause pain during physiotherapy in the postoperative period. Further repeat surgeries are required in patients due to hardware prominence ${ }^{[9]}$. However, due to use of standardised $3.5 \mathrm{~mm}$ precontoured locking plates, as put forth by the AO group, the incidence of hardware prominence was low. None of the patients in the present study underwent implant removal procedures.

Ulnar nerve injury is very common in distal humerus fractures. The time of injury can be at the time of trauma or during the surgical intervention. Trauma to the nerve can be due to the fracture fragments themselves or during the handling of the nerve during the surgery. Ulnar nerve transposition is an added step but has shown no benefit as reported by Sanchez-Sotelo et al. ${ }^{[4]}$. The olecranon osteotomy approach offers better visualisation of the ulnar nerve thus allowing the nerve to be identified, tagged and retracted before the beginning of the reduction of fracture fragments [11]. There was zero incidence of ulnar neuropathy in the present study.

Infections at the incision site both in the superficial and deep planes is a complication with distal end of humerus fractures. Adequate antibiotic coverage, thorough wash before wound closure, and exclusion of patients with Type 3 compound injuries by Gustilo-Anderson classification lead to zero incidence of infection in the present study.

There are two main approaches put forth for access to the distal humerus fracture namely, extensor mechanism-on method and the olecranon osteotomy approach. The extensoron mechanism approach preserves the neurovascular supply of the triceps and the anconeus but does not offer adequate visualisation of the fracture site. Further, the chances of triceps avulsion were reported to be higher. The olecranon osteotomy approach is tedious but offers complete visualisation of the fracture site. In 1969, the olecranon osteotomy approach was popularized by Cassebaum though MacAusland had described this technique as early as in 1915. There are several modifications to this technique, such as the chevron-shaped osteotomy, which is commonly advocated by the AO group ${ }^{[11]}$. The chevron osteotomy increases rotational and translational stability at the time of surgery and increases the contact area for achieving the bony union. Failure of bony union is a complication of the olecranon osteotomy approach. None of the cases performed with the trans olecranon approach, reported with non-union as a complication as observed by Ring et al. Gofton et al. recommended utilizing a contoured $3.5 \mathrm{~mm}$ reconstruction plate tofix the olecranon osteotomy, as it provided the most reproducible results in their series. Their study also reported a zero incidence of nonunion, and also no isolated procedures for hardware removal. Taking into account the additional financial burden put onto the patient with an additional implant, in our study, the 
olecranon osteotomy is fixed by two parallel K-wires passed obliquely into the proximal ulna through the anterior cortex, below the coronoid and fixed by tension band wiring method. The two tightening loops and bend wires are then pushed until they are buried under the triceps tendon. There was no incidence of non-union in our study with this method. Hence, the contoured plate to fix olecranon was not used.

\section{Conclusion}

The management of distal humerus fractures are often challenging owing to complex nature of fracture, with adequate congruity of fragments as a must to ensure acceptable range of motion. The key to successful treatment of these fractures is obtaining anatomic reduction with stable fixation to allow early range of motion. Performing anatomic reductions while minimizing soft tissue trauma will lead to improved patient outcomes while minimizing the complication rates. The posterior olecranon osteotomy approach gives excellent exposure of the distal articular surface of the humerus. Use of bicolumnar orthogonal plating is found to be successful method in obtaining the desired results if performed with expertise. The olecranon osteotomy is preferably fixed by two parallel $\mathrm{K}$-wires passed obliquely into the proximal ulnar anterior cortex below the coronoid and fixed by tension band wiring in figure of eight shape and bend wires that are pushed until they are buried under the triceps tendon.

\section{References}

1. Korner J, Lill H, Muller LP, Rommens PM, Schneider E, Linke B et al. The LCP-concept in the operative treatment of distal humerus fractures biological, biomechanical and surgical aspects. Injury. 2003; 34(Suppl 2):B20-30.

2. Galano GJ, Ahmad CS, Levine WN. Current treatment strategies for bicolumnar distal humerus fractures. J Am Acad Orthop Surg. 2010; 18:20-30.

3. Corradi A, Talamonti T, Cabitza P, Bottiglieri G, Secondi F. Innovative techniques for the osteosynthesis of distal humeral fractures. Injury. 2010; 41:1117-9.

4. Sanchez-Sotelo J. Distal humeral fractures: role of internal fixation and elbow arthroplasty. Instr Course Lect. 2012; 61:203-13

5. Yamaguchi K, Stein JA. Elbow arthroplasty for the treatment of bicolumn distal humeral fractures. Instr Course Lect. 2009; 58:529-33.

6. Pooley J, Salvador Carreno J. Total elbow joint replacement for fractures in the elderly Functional and radiological outcomes. Injury. 2015; 46(Suppl 5):S37-42.

7. Bryan RS, Morrey BF. Extensive posterior exposure of the elbow. ClinOrthopRelat Res. 1982; 166:188-92.

8. Green A. Open reduction and internal fixation with $90-90$ plating of bicolumn distal humeral fractures. Instr Course Lect. 2009; 58:515-9.

9. Abzug JM, Dantuluri PK. Use of orthogonal or parallel plating techniques to treat distal humerus fractures. Hand Clin. 2010; 26:411-21.

10. Leigey DF, Farrell DJ, Siska PA, Tarkin IS. Bicolumnar 90-90 plating of low-energy distal humeral fractures in the elderly patient. Geriatr Orthop Surg Rehabil. 2014; 5:1226.

11. Coles CP, Barei DP, Nork SE, Taitsman LA, Hanel DP, Bradford Henley M et al. The olecranon osteotomy: a sixyear experience in the treatment of intraarticular fractures of the distal humerus. J Orthop Trauma. 2006; 20:164-71.

12. Morrey BF, An KN. In: Morrey BF, editor. The elbow and its Disorders: Functional evaluation of the elbow. 3rd ed. Philadelphia: WB Saunders, 2000. p.82.

13. Turchin DC et al., Validity of Observer-Based Aggregate Scoring Systems as Descriptors of Elbow Pain, Function, and Disability, The Journal of Bone \& Joint Surgery, nr 80, 1998.p.154-62

14. Bryan RS, Morray BF. Extensive posterior exposure of the elbow. A tricpes sparing approach. ClinOrthopRelat Res. 1982; (166):188-92.

15. Babhulkar S, Pande KC, Babhulkar AS, Babhulkar SS. Primary reconstruction of comminutedintraarticular distal humerus fractures. International J Ortho Trauma. 1995; 5:170-4.

16. Robinson CM, Hill RM, Jacobs N, Dall G, CourtBrown CM. Adult distal humeral metaphyseal fractures: Epidemiology and results of treatment. J Orthop Trauma. 2003; 17:38-47.

17. McKee MD, Wilson TL, Winston L, Schemitsch EH, Richards RR. Functional outcome following surgical treatment of intra-articular distal humeral fractures through a posterior approach. J Bone Joint Surg Am. 2000; 82:1701-7.

18. Cobb TK, Morrey BF. Total elbow arthroplasty as primary treatment for distal humeral fractures in elderly patients. J Bone Joint Surg Am. 1997; 79-A:826-32.

19. Sanchez-Sotelo J, Torchia ME, O’Driscoll SW. Complex distal humeral fractures: Internal fixation with a principlebased parallel-plate technique. J Bone Joint Sur Am. 2007; 89:961-9.

20. Frankle MA, Herscovici D Jr, Dipasquale TG, Vasey MB, Sanders RW. A comparison of open reduction and internal fiation and Primary total elbow arthroplasty in the treatment of intrarticular distal humerus fractures in women older than age 65. J Orthop Trauma. 2003;17:47-9.

21. Jupiter JB, Neff U, Holzach P et al. Intercondylar fracture of the humerus. J Bone Joint Surg. 1985; 67:226-39.

22. McKee MD, Jupiter JB. A contemporary approach to the management of complex fractures of the distal humerus and their sequelae. Hand Clin. 1994; 10:479-94.

23. McKee MD, Jupiter JB, Toh CL et al. Reconstruction after malunion and nonunion of intra-articular fractures of the distal humerus. Methods and results in 13 adults. J Bone Joint Surg Br. 1994; 76:614-21.

24. Waddell JP, Hatch J, Richards RR. Supracondylar fractures of the humerus: Results of surgical treatment. J Trauma. 1988; 28:1615-21.

25. Kumar MN, Ravishankar MR, Manur R. Single locking compression plate fixation of extraarticular distal humeral fractures. J OrthopTraumatol. 2015; 16:99-104.

26. Shin SJ, Sohn HS, Do NH. A clinical comparison of two different double plating methods for intraarticular distal humerus fractures. Journal Shoulder Elbow Surg. 2010; 19:2-9.

27. Lan X, Zhang LH, Tao S, Zhang Q, Liang XD, Yuan BT et al. Comparative study of perpendicular versus parallel double plating methods for type $\mathrm{C}$ distal humeral fractures. Chin Med J (Engl). 2013; 126:2337-42.

28. Joshua M Abzug, Phani K Dantuluri. Use of orthogonal or parallel plating techniques to treat distal humerus fractures. Thomas Jefferson University Jefferson Digital Commons. 2010; 26(3):411-21

29. Jason M Erpelding, Adam Mailander, OTR/L Robin High, Matthew A Mormino, Edward V Fehringer. Outcomes following distal humeral fracture fixation with an extensor mechanism-on approach. the journal of bone and joint surgery; 2012; 94-a:548-53. 